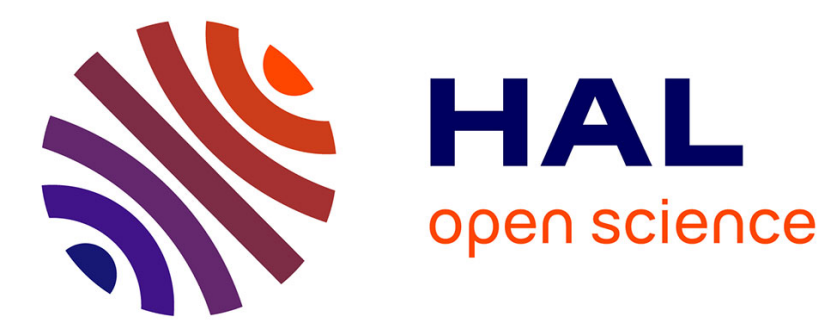

\title{
Reliable error estimation for Sobol' indices
}

Lluís Antoni Jiménez Rugama, Laurent Gilquin

\section{To cite this version:}

Lluís Antoni Jiménez Rugama, Laurent Gilquin. Reliable error estimation for Sobol' indices. Statistics and Computing, 2018, 28 (4), pp.725-738. 10.1007/s11222-017-9759-1 . hal-01358067v2

\section{HAL Id: hal-01358067 \\ https://hal.inria.fr/hal-01358067v2}

Submitted on 5 Jan 2017

HAL is a multi-disciplinary open access archive for the deposit and dissemination of scientific research documents, whether they are published or not. The documents may come from teaching and research institutions in France or abroad, or from public or private research centers.
L'archive ouverte pluridisciplinaire HAL, est destinée au dépôt et à la diffusion de documents scientifiques de niveau recherche, publiés ou non, émanant des établissements d'enseignement et de recherche français ou étrangers, des laboratoires publics ou privés. 


\title{
Reliable error estimation for Sobol’ indices
}

\author{
Lluís Antoni Jiménez Rugama · Laurent Gilquin
}

Received: date / Accepted: date

\begin{abstract}
In the field of sensitivity analysis, Sobol' indices are sensitivity measures widely used to assess the importance of inputs of a model to its output. The estimation of these indices is often performed trough Monte Carlo or quasiMonte Carlo methods. A notable method is the replication procedure that estimates first-order indices at a reduced cost in terms of number of model evaluations.

An inherent practical problem of this estimation is how to quantify the number of model evaluations needed to ensure that estimates satisfy a desired error tolerance. This paper addresses this challenge by proposing a reliable error bound for first-order and total effect Sobol' indices. Starting from the integral formula of the indices, the error bound is defined in terms of the discrete Walsh coefficients of the different integrands.

We propose a sequential estimation procedure of Sobol' indices using the error bound as a stopping criterion. The sequential procedure combines Sobol' sequences with either Saltelli's strategy to estimate both first-order and total effect indices, or the replication procedure to estimate only firstorder indices.
\end{abstract}

Keywords Sobol' index · error bound - sequential method . quasi-Monte Carlo

This work is supported by the CITiES project funded by the Agence Nationale de la Recherche (grant ANR-12-MONU-0020) and by the United States National Science Foundation (grant DMS-1522687).

Lluís Antoni Jiménez Rugama

Illinois Institute of Technology, Rettaliata Engineering Center, Room 208, $10 \mathrm{~W}$ 32st, Chicago, IL 60616

E-mail: ljimene1@hawk.iit.edu

Laurent Gilquin

Inria Grenoble - Rhône-Alpes, Inovallée, 655 avenue de l'Europe, 38330 Montbonnot

E-mail: gilquin.laurent@inria.fr
Mathematics Subject Classification (2000) 49Q12 . $62 \mathrm{~L} 12 \cdot 65 \mathrm{R} 10$

\section{Introduction}

Let $f$ represent a deterministic numerical model in $[0,1]^{d}$, $d \geqslant 1$. Sensitivity measures, also known as Sobol' indices, are used to assess which inputs of $f$ are influential for the output. The normalized indices are scalars between 0 and 1 whose values are interpreted as follows: the closer to 1 the more influential the index. Alternatively, they can be interpreted as the percentage of the variance explained by the inputs. Among all Sobol' indices one can distinguish firstorder and total effect indices. The first measure the effect of a single input, while the second measure the effect of a single input plus all its interactions with other inputs.

When dealing with complex numerical models, analytical expressions of Sobol' indices are often inaccessible. In such cases, one must rely on an estimation of these indices. The original estimation procedure is due to Sobol' [17]. However, this procedure requires several model evaluations which are usually expensive. Later on, Saltelli [15] proposed strategies to estimate sets of Sobol' indices at once through the use of a combinatorial formalisms. While elegant, these strategies still require a large number of model evaluations. A cost efficient alternative to estimate first-order indices was introduced in [10]. This alternative, called the replication procedure, has recently been further studied in [18] and generalized to the estimation of closed second-order indices.

A practical problem concerning the use of these methods is how to quantify the number of model evaluations required to ensure that Sobol' estimates are accurate enough. This article addresses this challenge by proposing a reliable error bound for Sobol' indices based on digital sequences. The error bound is defined in terms of the discrete Walsh 
coefficients of the integrands involved in the Sobol' indices definition. We propose a sequential estimation procedure of Sobol' indices using the error bound as our stopping criterion. The procedure operates under the assumption that all integrands lie inside a particular cone of functions (see [4]).

Firstly, Section 2 introduces Sobol' indices and reviews both Saltelli's strategy to estimate first-order and total effect Sobol' indices, and the replication procedure. Our main contribution is detailed in Section 3. There, we review the construction of the error bound proposed in [4] for the estimation of integrals based on digital sequences, and then we generalize it for Sobol' indices. Section 4 is devoted to analyze the cost in terms of model evaluations of our sequential estimation algorithm. It combines the error bound in Section 3 and either one of the two estimation procedures of Section 2. We also discuss a potential improvement to estimate small first-order indices according to [14]. Finally, examples and illustrations of our procedure are presented in Section 5.

\section{Background on Sobol' indices}

\subsection{Definition of Sobol' indices}

Denote by $\mathbf{x}=\left(x_{1}, \ldots, x_{d}\right)$ the vector of inputs of $f$. We assume that $f$ is in some subset of $\in \mathbb{L}^{2}\left([0,1]^{d}\right)$ for which $f(\mathbf{x})$ is defined for all $\mathbf{x} \in[0,1]^{d}$, and $\mathscr{D}=\{1, \ldots, d\}$ the set of dimension indexes. Let $u$ be a subset of $\mathscr{D},-u$ its complement and $|u|$ its cardinality. Then, $\mathbf{x}_{u}$ represents a point in $[0,1]^{|u|}$ with components $x_{j}, j \in u$. Given two points $\mathbf{x}$ and $\mathbf{x}^{\prime}$, the hybrid point $\mathbf{w}=\left(\mathbf{x}_{u}: \mathbf{x}^{\prime}{ }_{-u}\right)$ is defined as $w_{j}=x_{j}$ if $j \in u$ and $w_{j}=x_{j}^{\prime}$ if $j \notin u$.

The uncertainty on $\mathbf{x}$ is modeled by a uniform random vector, namely $\mathbf{x} \stackrel{\text { iid }}{\sim} \mathscr{U}\left([0,1]^{d}\right)$. The Hoeffding decomposition $[6,17]$ of $f$ is:

$f(\mathbf{x})=f_{\varnothing}+\sum_{u \subseteq \mathscr{D}, u \neq \varnothing} f_{u}(\mathbf{x})$,

where:

$$
\begin{aligned}
f_{\varnothing} & =\mathbb{E}[f(\mathbf{x})]=\mu, \\
f_{u}(\mathbf{x}) & =\int_{[0,1]^{|u|}} f(\mathbf{x}) d \mathbf{x}_{-u}-\sum_{v \subset u} f_{v}(\mathbf{x}) .
\end{aligned}
$$

Due to orthogonality, the variance of equation (1) leads to the variance decomposition of $f$ :

$\sigma^{2}=\operatorname{Var}[f(\mathbf{x})]=\sum_{u \subseteq \mathscr{D}, u \neq \varnothing} \sigma_{u}^{2}$, with $\sigma_{u}^{2}=\int_{[0,1]^{|u|}} f_{u}(\mathbf{x})^{2} d \mathbf{x}_{u}$.

From this decomposition, one can define the following two quantities:

$\underline{\tau}_{u}^{2}=\sum_{v \subseteq u} \sigma_{v}^{2}, \quad \bar{\tau}_{u}^{2}=\sum_{v \cap u \neq \varnothing} \sigma_{v}^{2}, \quad u \subsetneq \mathscr{D}$.
These two quantities $\underline{\tau}_{u}^{2}$ and $\bar{\tau}_{u}^{2}$ measure the importance of variables $\mathbf{x}_{u}: \tau_{u}^{2}$ quantifies the main effect of $\mathbf{x}_{u}$, that is the effect of all interactions between variables in $\mathbf{x}_{u}$, and $\bar{\tau}_{u}^{2}$ quantifies the main effect of $\mathbf{x}_{u}$ plus the effect of all interactions between variables in $\mathbf{x}_{u}$ and variables in $\mathbf{x}_{-u}$.

Both $\tau_{u}^{2}$ and $\bar{\tau}_{u}^{2}$ satisfy the following relations: $0 \leqslant \tau_{u}^{2} \leqslant$ $\bar{\tau}_{u}^{2}$ and $\underline{\tau}_{u}^{2}=\sigma^{2}-\bar{\tau}_{-u}^{2}$. These two measures are commonly found in the literature in their normalized form: $\underline{S}_{u}=\tau_{u}^{2} / \sigma^{2}$ is the closed $|u|$-order Sobol' index for inputs $u$, while $\bar{S}_{u}=$ $\bar{\tau}_{u}^{2} / \sigma^{2}$ is the total effect Sobol' index of order $|u|$.

In our framework, we are only interested in single input indices, namely $|u|=1$. The computation of the normalized indices is performed based on the following integral formulas for their numerators:

$$
\begin{aligned}
\underline{\tau}_{u}^{2} & =\int_{[0,1]^{2 d}}\left(f(\mathbf{x})-f\left(\mathbf{x}_{u}: \mathbf{x}_{-u}^{\prime}\right)\right) f\left(\mathbf{x}^{\prime}\right) d \mathbf{x} d \mathbf{x}^{\prime}, \\
\bar{\tau}_{u}^{2} & =\frac{1}{2} \int_{[0,1]^{d+1}}\left(f\left(\mathbf{x}^{\prime}\right)-f\left(\mathbf{x}_{u}: \mathbf{x}^{\prime}{ }_{-u}\right)\right)^{2} d \mathbf{x}_{u} d \mathbf{x}^{\prime}, u \in \mathscr{D},
\end{aligned}
$$

while variance and mean of $f$ are evaluated as:

$$
\begin{aligned}
\sigma^{2} & =\int_{[0,1]^{d}} f(\mathbf{x})^{2} d \mathbf{x}-\mu^{2}, \\
\mu & =\int_{[0,1]^{d}} f(\mathbf{x}) d \mathbf{x},
\end{aligned}
$$

Usually the complexity of $f$ causes the solution of integrals (2), (3) and (4) to be intractable. In such cases, one can instead estimate these quantities.

\subsection{Estimation of Sobol' indices}

In this section we review two Monte Carlo procedures for the estimation of Sobol' indices. A design is a point set $\mathscr{P}=\left\{\mathbf{x}_{i}\right\}_{i=0}^{n-1}$ where each point is obtained by sampling each variable $x_{j} n$ times. Each row of the design is a point $\mathbf{x}_{i}$ in $[0,1]^{d}$ and each column of the design refers to samples of a variable $x_{j}$. Consider $\mathscr{P}=\left\{\mathbf{x}_{i}\right\}_{i=0}^{n-1}$ and $\mathscr{P}^{\prime}=\left\{\mathbf{x}_{i}^{\prime}\right\}_{i=0}^{n-1}$ two designs where $\left(\mathbf{x}_{i}, \mathbf{x}_{i}^{\prime}\right) \stackrel{\text { iid }}{\sim} \mathscr{U}\left([0,1]^{2 d}\right)$. One way to estimate the two quantities (2) and (3) is via:

$$
\begin{aligned}
& \widehat{\tau_{u}^{2}}=\frac{1}{n} \sum_{i=0}^{n-1}\left(f\left(\mathbf{x}_{i}\right)-f\left(\mathbf{x}_{i, u}: \mathbf{x}_{i,-u}^{\prime}\right)\right) f\left(\mathbf{x}_{i}^{\prime}\right), \\
& \widehat{\bar{\tau}_{u}^{2}}=\frac{1}{2 n} \sum_{i=0}^{n-1}\left(f\left(\mathbf{x}_{i}^{\prime}\right)-f\left(\mathbf{x}_{i, u}: \mathbf{x}_{i,-u}^{\prime}\right)\right)^{2}, \quad u \in \mathscr{D},
\end{aligned}
$$

using for $\sigma^{2}$ :

$\widehat{\sigma}^{2}=\frac{1}{n} \sum_{i=0}^{n-1} f\left(\mathbf{x}_{i}\right)^{2}-\widehat{\mu}^{2}$, with $\widehat{\mu}=\frac{1}{n} \sum_{i=0}^{n-1} f\left(\mathbf{x}_{i}\right)$. 
Then, the Sobol' indices estimators are:

$\widehat{S}_{u}=\widehat{\tau}_{u}^{2} / \hat{\sigma}^{2}, \quad \widehat{\bar{S}}_{u}=\widehat{\bar{\tau}}_{u}^{2} / \hat{\sigma}^{2}$.

Using this method, the estimation of a single pair $\left(\widehat{S}_{u}, \hat{\bar{S}}_{u}\right)$ requires $3 n$ evaluations of the model $f$. Thus, for all first order and total effect indices, one would need 3 nd model evaluations. Using a combinatorial formalism, in [15] Saltelli proposes the following estimation strategy:

Theorem 1 The $d+2$ designs $\left\{\mathbf{x}_{i, u}: \mathbf{x}_{i,-u}^{\prime}\right\}_{i=0}^{n-1}$ constructed for $u \in\{\varnothing,\{1\}, \ldots,\{d\}, \mathscr{D}\}$ allow to estimate all first-order and total effect Sobol' indices at a cost of $n(d+2)$ evaluations of the model.

The main idea of the theorem is that there is no need to reevaluate $f\left(\mathbf{x}_{i}\right)$ and $f\left(\mathbf{x}_{i}^{\prime}\right)$ for each $u$. Hence, we can simply evaluate $f\left(\mathbf{x}_{i}\right) n$ times, $f\left(\mathbf{x}_{i}^{\prime}\right) n$ times, and $f\left(\mathbf{x}_{i, u}: \mathbf{x}_{i,-u}^{\prime}\right) n d$ times, which accounts for the $n(d+2)$ evaluations.

The $d+2$ designs of Theorem 1 are obtained by substituting columns of $\mathscr{P}$ for columns of $\mathscr{P}^{\prime}$ accordingly to $u$. While elegant, this approach requires a number of model evaluations that grows linearly with respect to the input space dimension.

A more efficient alternative to evaluate all first-order indices was proposed in [10] and only requires $2 n$ model evaluations. This alternative relies on the construction of two replicated designs. The notion of replicated designs was first introduced by McKay through his replicated Latin Hypercubes in [11]. In order to apply this definition to other types of points, in [3] we generalized this notion to the following.

Definition 1 Let $\mathscr{P}=\left\{\mathbf{x}_{i}\right\}_{i=0}^{n-1}$ and $\mathscr{P}^{\prime}=\left\{\mathbf{x}_{i}^{\prime}\right\}_{i=0}^{n-1}$ be two point sets in $[0,1]^{d}$. Let $\mathscr{P}^{u}=\left\{\mathbf{x}_{i, u}\right\}_{i=0}^{n-1}\left(\right.$ resp. $\left.\mathscr{P}^{\prime u}\right), u \subsetneq \mathscr{D}$, denote the subset of dimensions of $\mathscr{P}$ (resp. $\mathscr{P}^{\prime}$ ) indexed by $u$. We say that $\mathscr{P}$ and $\mathscr{P}^{\prime}$ are two replicated designs of order $a \in\{1, \ldots, d-1\}$ if for any $u \subsetneq \mathscr{D}$ such that $|u|=a, \mathscr{P}^{u}$ and $\mathscr{P}^{\prime u}$ are the same point set in $[0,1]^{a}$. We define by $\pi_{u}$ the permutation that rearranges the rows of $\mathscr{P}^{\prime u}$ into $\mathscr{P}^{u}$.

The method introduced in [10] allows to estimate all firstorder Sobol' indices with only two replicated designs of order 1 . The key point of this method is to use the permutations resulting from the structure of the two replicated designs to mimic the hybrid points in equation (5).

More precisely, let $\mathscr{P}=\left\{\mathbf{x}_{i}\right\}_{i=0}^{n-1}$ and $\mathscr{P}^{\prime}=\left\{\mathbf{x}_{i}^{\prime}\right\}_{i=0}^{n-1}$ be two replicated designs of order 1 . Denote by $\left\{y_{i}\right\}_{i=0}^{n-1}=$ $\left\{f\left(\mathbf{x}_{i}\right)\right\}_{i=0}^{n-1}$ and $\left\{y_{i}^{\prime}\right\}_{i=0}^{n-1}=\left\{f\left(\mathbf{x}_{i}^{\prime}\right)\right\}_{i=0}^{n-1}$ the two sets of model evaluations obtained with $\mathscr{P}$ and $\mathscr{P}^{\prime}$. From Definition 1, we know that $\mathbf{x}_{\pi_{u}(i), u}^{\prime}=\mathbf{x}_{i, u}$. Then,

$$
\begin{aligned}
y_{\pi_{u}(i)}^{\prime} & =f\left(\mathbf{x}_{\pi_{u}(i), u}^{\prime}: \mathbf{x}_{\pi_{u}(i),-u}^{\prime}\right) \\
& =f\left(\mathbf{x}_{i, u}: \mathbf{x}_{\pi_{u}(i),-u}^{\prime}\right) .
\end{aligned}
$$

Hence, each $\tau_{u}^{2}$ can be estimated via formula (5) by using $y_{\pi_{u}(i)}^{\prime}$ instead of $f\left(\mathbf{x}_{i, u}: \mathbf{x}_{i,-u}^{\prime}\right)$ without requiring further model evaluations for each $u$. This estimation method has been studied deeply and generalized in Tissot et al. [18] to the case of closed second-order indices. In the following we will refer to this method as the replication procedure.

\subsection{Towards a reliable estimation}

The aim of this article is to propose a sequential procedure to estimate first-order and total effect Sobol' indices. A practical problem concerning the estimation of these indices is how large to choose the number of evaluations to ensure that Sobol' estimates are accurate enough. Asymptotical results show that Sobol' estimates are normally distributed ([7, Proposition 2.2], [18, Proposition 3.5]). As a consequence, errors can be estimated through confidence intervals. However, these error estimates are only guaranteed asymptotically as the number of model evaluations goes to infinity.

Additional sequential procedures are the replicated procedure and McKay's procedure respectively proposed in [2] and [19]. Nevertheless, in those two cases, the stopping criterion is a purely empirical quantity of interest, built directly upon the estimates. Such stopping criteria often involve hyper-parameters that are difficult to tweak but more importantly, fail to guarantee any error bound on the estimates.

Our sequential procedure stands apart from others since it proposes a robust stopping criterion, not costly to compute. This criterion is an error bound based on the Walsh series decomposition of the integrands in (2), (3) and (4), and exploits the group properties of digital nets. As such, our procedure relies on an iterative construction of Sobol' sequences. This construction is performed accordingly to the multiplicative approach presented in [3].

The description of the error bound is introduced in the following section and our sequential procedure is detailed in Section 4.

\section{Reliable error bound for Sobol' indices}

We start by reviewing the construction of the error bound proposed in [4] for the estimation of $d$-dimensional integrals. Then, we present an extension of this error bound for normalized Sobol' indices. This extension is built upon the integral formula of a Sobol' index.

\subsection{Reliable integral estimation using digital sequences}

We assume we have an embedded sequence of digital nets in base $b$ as in [4, Sec. 2-3],

$$
\mathscr{P}_{0}=\{\mathbf{0}\} \subset \cdots \subset \mathscr{P}_{m}=\left\{\mathbf{x}_{i}\right\}_{i=0}^{b^{m}-1} \subset \cdots \subset \mathscr{P}_{\infty}=\left\{\mathbf{x}_{i}\right\}_{i=0}^{\infty} .
$$


Each $\mathscr{P}_{m}$ has a group structure under the digitwise addition: $\mathbf{x} \oplus \mathbf{t}=\left(\sum_{\ell=1}^{\infty}\left[\left(x_{j \ell}+t_{j \ell}\right) \bmod b\right] b^{-\ell}(\bmod 1)\right)_{j=1}^{d}$,

where $x_{j \ell}$ and $t_{j \ell}$ are the $b$-adic decompositions of the $j^{\text {th }}$ component of points $\mathbf{x}$ and $\mathbf{t}$.

To relate the group structure of $\mathscr{P}_{m}$ with the integration error, we introduce the dual net which establishes the relationship between any digital net and the wavenumber space of non-negative integers $\mathbb{N}_{0}^{d}$. A dual net is

$$
\begin{gathered}
\mathscr{P}_{m}^{\perp}=\left\{\mathbf{k} \in \mathbb{N}_{0}^{d}:\langle\mathbf{k}, \mathbf{x}\rangle=0, \mathbf{x} \in \mathscr{P}_{m}\right\}, \\
\langle\mathbf{k}, \mathbf{x}\rangle=\sum_{j=1}^{d} \sum_{\ell=0}^{\infty} k_{j \ell} x_{j, \ell+1} \quad(\bmod b),
\end{gathered}
$$

and inherits the same embedded structure as for digital nets, $\mathscr{P}_{0}^{\perp}=\mathbb{N}_{0}^{d} \supset \cdots \supset \mathscr{P}_{\infty}^{\perp}=\{\mathbf{0}\}$.

As shown in [4, Sec. 3], the group structure of digital nets guarantees the property below affecting any Walsh basis $\varphi_{\mathbf{k}}(\mathbf{x})=\mathrm{e}^{2 \pi \sqrt{-1}\langle\mathbf{k}, \mathbf{x}\rangle / b}$,

$\frac{1}{b^{m}} \sum_{\mathbf{x} \in \mathscr{P}_{m}} \varphi_{\mathbf{k}}(\mathbf{x})= \begin{cases}1, & \mathbf{k} \in \mathscr{P}_{m}^{\perp}, \\ 0, & \mathbf{k} \notin \mathscr{P}_{m}^{\perp} .\end{cases}$

Therefore, considering the Walsh decomposition of any $f \in \mathbb{L}^{2}\left([0,1]^{d}\right):$

$f(\mathbf{x})=\sum_{\mathbf{k} \in \mathbb{N}_{0}^{d}} \hat{f}_{\mathbf{k}} \varphi_{\mathbf{k}}(\mathbf{x})$,

and,

$I=\int_{[0,1]^{d}} f(\mathbf{x}) d \mathbf{x}$

property (10) leads to

$$
\begin{aligned}
\left|I-\frac{1}{b^{m}} \sum_{\mathbf{x} \in \mathscr{P}_{m}} f(\mathbf{x})\right| & =\left|\hat{f}_{\mathbf{0}}-\frac{1}{b^{m}} \sum_{\mathbf{x} \in \mathscr{P}_{m}} \sum_{\mathbf{k} \in \mathbb{N}_{0}^{d}} \hat{f}_{\mathbf{k}} \varphi_{\mathbf{k}}(\mathbf{x})\right|, \\
& =\left|\sum_{\mathbf{k} \in \mathscr{P} \perp \backslash\{\mathbf{0}\}} \hat{f}_{\mathbf{k}}\right| \\
& \leqslant \sum_{\mathbf{k} \in \mathscr{P} \frac{\perp}{m} \backslash\{\mathbf{0}\}}\left|\hat{f}_{\mathbf{k}}\right| .
\end{aligned}
$$

Based on the size of $\left|\hat{f}_{\mathbf{k}}\right|$ and the structure of dual nets (9), in [4, Sec. 4.1] we proposed an ordering of the wavenumbers $\mathbf{k}(\cdot): \mathbb{N}_{0} \rightarrow \mathbb{N}_{0}^{d}$. Defining $\hat{f}_{\kappa}=\hat{f}_{\mathbf{k}(\kappa)}$, the error bound (11) becomes,

$\left|I-\frac{1}{b^{m}} \sum_{\mathbf{x} \in \mathscr{P}_{m}} f(\mathbf{x})\right| \leqslant \sum_{\lambda=1}^{\infty}\left|\hat{f}_{\lambda b^{m}}\right|$.

Nonetheless, because the knowledge of the Walsh coefficients $\hat{f}_{\kappa}$ is not assumed, we will estimate them using the fast transform obtained with the precomputed function values, and refer to them as $\widetilde{f}_{m, \kappa}$,

$\widetilde{f}_{m, \kappa}=\tilde{f}_{m, \mathbf{k}(\kappa)}=\frac{1}{b^{m}} \sum_{\mathbf{x} \in \mathscr{P}_{m}} f(\mathbf{x}) \overline{\varphi_{\mathbf{k}(\kappa)}(\mathbf{x})}$.

Note that for function values evaluated at $\mathscr{P}_{m}$, one only generates $b^{m}$ discrete Walsh coefficients $\widetilde{f}_{m, \kappa}$.

For $\ell, m \in \mathbb{N}_{0}$ and $\ell \leqslant m$ we introduce the following notation,

$$
\begin{aligned}
S_{m}(f) & =\sum_{\kappa=\left\lfloor b^{m-1}\right\rfloor}^{b^{m}-1}\left|\hat{f}_{\kappa}\right|, \hat{S}_{\ell, m}(f)=\sum_{\kappa=\left\lfloor b^{\ell-1}\right\rfloor}^{b^{\ell}-1} \sum_{\lambda=1}^{\infty}\left|\hat{f}_{\kappa+\lambda b^{m}}\right|, \\
\check{S}_{m}(f) & =\widehat{S}_{0, m}(f)+\cdots+\widehat{S}_{m, m}(f)=\sum_{\kappa=b^{m}}^{\infty}\left|\hat{f}_{\kappa}\right|, \\
\widetilde{S}_{\ell, m}(f) & =\sum_{\kappa=\left\lfloor b^{\ell-1}\right\rfloor}^{b^{\ell}-1}\left|\tilde{f}_{m, \kappa}\right| .
\end{aligned}
$$

Finally, we define the set of functions $\mathscr{C}$,

$$
\begin{array}{r}
\mathscr{C}:=\left\{f \in \mathbb{L}^{2}\left([0,1]^{d}\right): \widehat{S}_{\ell, m}(f) \leqslant \widehat{\omega}(m-\ell) \check{S}_{m}(f), \quad \ell \leqslant m,\right. \\
\left.\check{S}_{m}(f) \leqslant \stackrel{\omega}{ }(m-\ell) S_{\ell}(f), \quad \ell_{*} \leqslant \ell \leqslant m\right\} .
\end{array}
$$

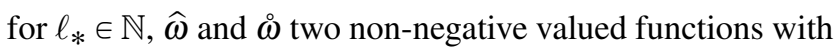
$\lim _{m \rightarrow \infty} \stackrel{\circ}{\omega}(m)=0$, and such that $\widehat{\omega}(r) \stackrel{\circ}{\omega}(r)<1$ for some fixed $r \in \mathbb{N}$.

In $[4$, Sec. 4.2] it is shown that for any $f \in \mathscr{C}$,

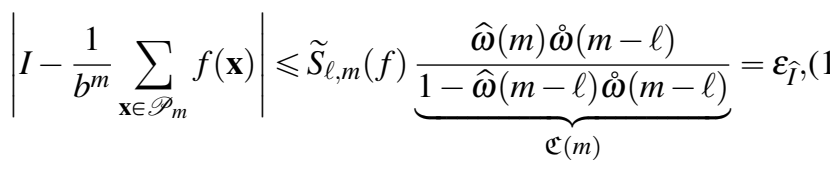

where one may increase $m$ until the error bound $\varepsilon_{I}$ is small enough.

Details concerning the algorithm, the mapping of the wavenumber space, or the meaning and properties of $\mathscr{C}$, are provided in [4].

For our problem, only Sobol' sequences [16] have been considered. Their major interest comes from their fast and easy implementation. Further details concerning Sobol' sequences can be found in $[9,12]$.

\subsection{Extension to Sobol' indices}

The idea here is to extend the definition of the error bound (14) to Sobol' indices. To achieve this goal, we consider the two integral formulas of the first-order and total effect 
Sobol' indices with $|u|=1$ :

$$
\begin{aligned}
& \underline{S}_{u}(\mathbf{I})=\frac{\int_{[0,1)^{2 d}}\left(f(\mathbf{x})-f\left(\mathbf{x}_{u}: \mathbf{x}^{\prime}{ }_{-u}\right)\right) f\left(\mathbf{x}^{\prime}\right) d \mathbf{x} d \mathbf{x}^{\prime}}{\int_{[0,1)^{d}} f(\mathbf{x})^{2} d \mathbf{x}-\left(\int_{[0,1)^{d}} f(\mathbf{x}) d \mathbf{x}\right)^{2}}, \\
& \underline{S}_{u}(\mathbf{I})=\frac{I_{1}}{I_{3}-\left(I_{4}\right)^{2}}, \\
& \bar{S}_{u}(\mathbf{I})=\frac{\frac{1}{2} \int_{[0,1)^{d+1}}\left(f\left(\mathbf{x}^{\prime}\right)-f\left(\mathbf{x}_{u}: \mathbf{x}^{\prime}{ }_{-u}\right)\right)^{2} d \mathbf{x}_{u} d \mathbf{x}^{\prime}}{\int_{[0,1)^{d}} f(\mathbf{x})^{2} d \mathbf{x}-\left(\int_{[0,1)^{d}} f(\mathbf{x}) d \mathbf{x}\right)^{2}}, \\
& \bar{S}_{u}(\mathbf{I})=\frac{I_{2}}{I_{3}-\left(I_{4}\right)^{2}},
\end{aligned}
$$

where $\mathbf{I}=\left(I_{1}, I_{2}, I_{3}, I_{4}\right)$ is a vector of integral values. Indices $\underline{S}_{u}(\mathbf{I})$ and $\bar{S}_{u}(\mathbf{I})$ are defined as functions over the vector $\mathbf{I}$. If we estimate $\mathbf{I}$ by $\widehat{\mathbf{I}}$ with vector of error bounds $\varepsilon_{\hat{\mathbf{I}}}=$ $\left(\varepsilon_{\widehat{I}_{1}}, \varepsilon_{\widehat{I}_{2}}, \varepsilon_{\widehat{I}_{3}}\right.$,

$\left.\varepsilon_{\widehat{I}_{4}}\right)$ according to (14), we know that $\mathbf{I} \in B_{\varepsilon_{\hat{\mathbf{I}}}}(\widehat{\mathbf{I}})=\left[\widehat{\mathbf{I}}-\varepsilon_{\hat{\mathbf{I}}}, \widehat{\mathbf{I}}+\right.$ $\left.\varepsilon_{\hat{\mathbf{I}}}\right]$.

Then, as an alternative to the common Sobol' indices estimators (8), we can define the following two estimators with their respective error bounds:

$$
\begin{aligned}
& \widehat{S}_{u}=\frac{1}{2}\left(\min \left(\max _{\mathbf{I} \in B_{\tilde{\varepsilon}_{\mathbf{I}}}(\hat{\mathbf{I}})} \underline{S}_{u}(\mathbf{I}), 1\right)+\max \left(\min _{\mathbf{I} \in B_{\varepsilon_{\hat{\mathbf{I}}}}(\hat{\mathbf{I}})} \underline{S}_{u}(\mathbf{I}), 0\right)\right) \\
& \varepsilon_{\widehat{\underline{S}}_{u}}=\frac{1}{2}\left(\min \left(\max _{\mathbf{I} \in B_{\varepsilon_{\hat{\mathbf{I}}}}(\widehat{\mathbf{I}})} \underline{S}_{u}(\mathbf{I}), 1\right)-\max \left(\min _{\mathbf{I} \in B_{\widehat{\varepsilon}_{\hat{\mathbf{I}}}}(\widehat{\mathbf{I}})} \underline{S}_{u}(\mathbf{I}), 0\right)\right)
\end{aligned}
$$

and,

$$
\begin{aligned}
\widehat{\bar{S}}_{u} & =\frac{1}{2}\left(\min \left(\max _{\mathbf{I} \in B_{\varepsilon_{\hat{\mathbf{I}}}}(\widehat{\mathbf{I}})} \bar{S}_{u}(\mathbf{I}), 1\right)+\max \left(\min _{\mathbf{I} \in B_{\tilde{\varepsilon}_{\hat{\mathbf{I}}}}(\widehat{\mathbf{I}})} \bar{S}_{u}(\mathbf{I}), 0\right)\right) \\
\varepsilon_{\hat{\bar{S}}_{u}} & =\frac{1}{2}\left(\min \left(\max _{\mathbf{I} \in B_{\tilde{\varepsilon}_{\mathbf{I}}}(\widehat{\mathbf{I}})} \bar{S}_{u}(\mathbf{I}), 1\right)-\max \left(\min _{\mathbf{I} \in B_{\tilde{\varepsilon}_{\mathbf{I}}}(\widehat{\mathbf{I}})} \bar{S}_{u}(\mathbf{I}), 0\right)\right)
\end{aligned}
$$

Because numerator and denominator are both known to be positive, maximizing $\underline{S}_{u}(\mathbf{I})$ (resp. $\left.\bar{S}_{u}(\mathbf{I})\right)$ is done through maximizing the numerator $I_{1}$ (resp. $I_{2}$ ) and minimizing the denominator $I_{3}-I_{4}^{2}$. Analogously, to minimize $\underline{S}_{u}(\mathbf{I})$ (resp. $\bar{S}_{u}(\mathbf{I})$ ) one minimizes the numerator $I_{1}$ (resp. $I_{2}$ ) and maximizes the denominator $I_{3}-I_{4}^{2}$. As an example, Figure 1 illustrates the region of possible values of $\underline{S}_{2}(\mathbf{I})$ given the true value of $I_{1}$ for the test function of Bratley et al. [1]. This function is further described in Section 5.

Under the assumption that each integrand in $\underline{S}_{u}$ and $\bar{S}_{u}$ lies inside $\mathscr{C}$, these new estimators satisfy:

$$
\underline{S}_{u} \in\left[\underline{\hat{S}}_{u}-\varepsilon_{\underline{\hat{S}}_{u}}, \widehat{\underline{S}}_{u}+\varepsilon_{\underline{S}_{u}}\right], \quad \bar{S}_{u} \in\left[\hat{\bar{S}}_{u}-\varepsilon_{\hat{\bar{S}}_{u}}, \hat{\bar{S}}_{u}+\varepsilon_{\widehat{\bar{S}}_{u}}\right] .
$$

For the rest of the paper, we will consider $\underline{\widehat{S}}_{u}$ and $\widehat{\bar{S}}_{u}$ as defined in formulas (15) and (16).
Fig. 1 The delimited region on the figure represents the values $\underline{S}_{2}$ in $\mathbf{I} \in B_{(0,0,0.05,0.1)}(\widehat{\mathbf{I}})$ for the Bratley et al. function. There, $\underline{S}_{2}^{\min }=$ $\max \left(\min _{\mathbf{I} \in B_{\varepsilon_{\mathbf{I}}}(\widehat{\mathbf{I}})} \underline{S}_{2}(\mathbf{I}), 0\right)$ and $\underline{S}_{2}^{\max }=\min \left(\max _{\mathbf{I} \in B_{\varepsilon_{\mathbf{I}}}(\widehat{\mathbf{I}})} \underline{S}_{2}(\mathbf{I}), 1\right)$, and $\underline{\hat{S}}_{2}=\left(\underline{S}_{2}^{\min }+\underline{S}_{2}^{\max }\right) / 2$.

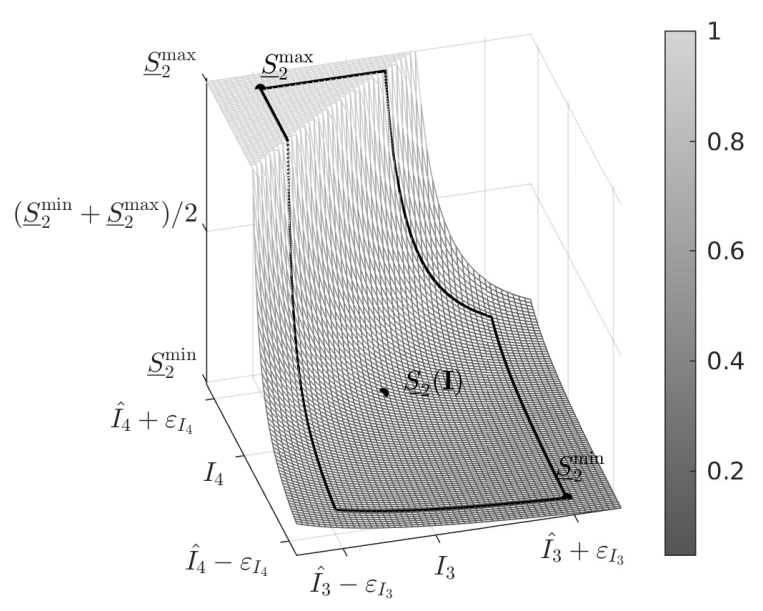

\section{Sequential estimation procedure}

The sequential estimation procedure we propose combines error bounds $\varepsilon_{\widehat{S}_{u}}$ and $\varepsilon_{\widehat{\bar{S}}_{u}}$ presented in the previous section with either one of the two estimation strategies of Section 2.2: Saltelli's strategy and the replication procedure.

We start by detailing our procedure under the form of an algorithm. Then, we discuss a possible improvement by considering a new estimator introduced in [14] for the estimation of first-order indices.

\subsection{Sequential algorithm and cost}

Algorithm 1 summarizes the main steps of our sequential procedure. First, one must fix the tolerance $\varepsilon>0$ at which Sobol' indices must be estimated, and set $m=\ell_{*}+r$, where $\ell_{*}$ and $r$ are the two hyper-parameters defined in (13). Then, one must construct the two designs $\mathscr{P}_{m}=\left\{\mathbf{x}_{i}\right\}_{i=0}^{2^{m}-1}$ and $\mathscr{P}_{m}^{\prime}=$ $\left\{\mathbf{x}_{i}^{\prime}\right\}_{i=0}^{2^{m}-1}$ at Step 5 according to the multiplicative approach detailed in [3]. The choice of $\ell_{*}$ must be large enough to ensure that each integrand of $\underline{S}_{u}$ and $\bar{S}_{u}$ (Section 3.2) is in $\mathscr{C}$ (see (13)).

Under this construction, $\mathscr{P}_{m}$ and $\mathscr{P}_{m}^{\prime}$ correspond to the first $2^{m}$ points of two independent Sobol' sequences. Furthermore, they possess the structure of two replicated designs of order 1 , as shown in [3]. In addition, $\mathscr{P}_{m}$ and $\mathscr{P}_{m}^{\prime}$ can also be scrambled as long as both point sets share the same scrambling dimensionwise. 
The sets $\mathscr{P}_{m}$ and $\mathscr{P}_{m}^{\prime}$ can be used with Saltelli's strategy to estimate all first-order indices and total effect Sobol' indices. This option is referred as Variant $A$ in Algorithm 1.

Alternatively, $\mathscr{P}_{m}$ and $\mathscr{P}_{m}^{\prime}$ can be used with the replication procedure to estimate all first-order Sobol' indices. This option is referred as Variant $B$ in Algorithm 1 .

In both cases we always check if the respective error bounds $\varepsilon_{\widehat{S}_{u}}$ and $\varepsilon_{\hat{\bar{S}}_{u}}$ are lower than the tolerance $\varepsilon$. For Variant $A$ the stopping criterion is $\varepsilon_{\widehat{S}_{u}} \leqslant \varepsilon$ and $\varepsilon_{\hat{\bar{S}}_{u}} \leqslant \varepsilon$ for all $u \in \mathscr{D}$. For Variant $B$ it is $\varepsilon_{\widehat{S}_{u}} \leqslant \varepsilon$ for all $u \in \mathscr{D}$.

If the stopping criterion is satisfied, the algorithm stops and Sobol' estimates are returned. Otherwise, $m$ is incremented by one to perform a new estimation.

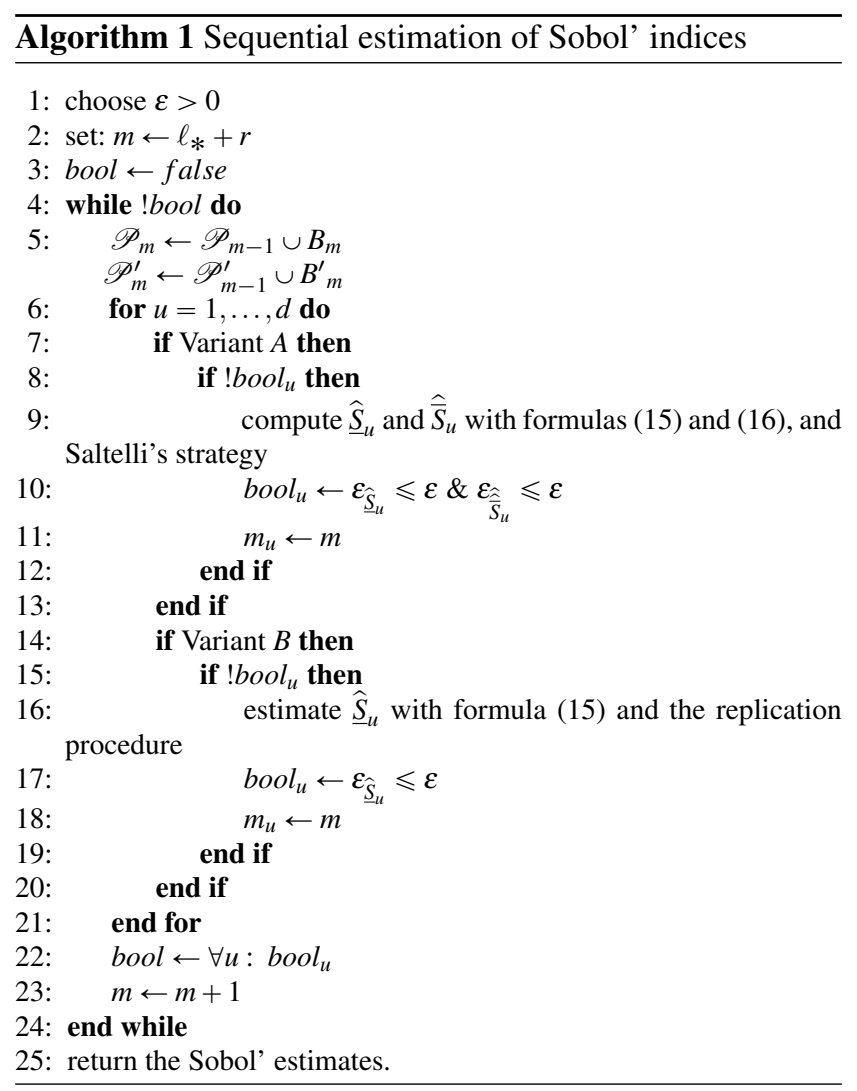

The cost of our algorithm, in terms of model evaluations, varies whether Variant $A$ or Variant $B$ is selected. To discuss this cost we note by $m^{\star}$ the ending iteration. If Variant $A$ is selected, the cost of our algorithm is:

$\sum_{u \in \mathscr{D}} 2^{m_{u}}+2 \times 2^{m^{\star}}, \quad m^{\star}=\max _{u \in \mathscr{D}} m_{u}$

where:

- $2^{m_{u}}$ is the number of evaluations $f\left(\mathbf{x}_{i, u}: \mathbf{x}_{i,-u}^{\prime}\right)$ used to estimate both the first-order index $\underline{S}_{u}$ and the total effect index $\bar{S}_{u}$,
- $2 \times 2^{m^{\star}}$ is the number of evaluations $f\left(\mathbf{x}_{i}\right)$ and $f\left(\mathbf{x}_{i}^{\prime}\right)$ used in the estimation of each first-order and total effect indices.

If all $m_{u}$ are equal, the cost of Variant $A$ becomes $2^{m^{\star}}(d+2)$ and we recover the cost specified in Theorem 1 with $n=2^{m^{\star}}$.

If Variant $B$ is selected, the cost of our algorithm equals $2 \times 2^{m^{\star}}$. This cost corresponds to the one of the replication procedure introduced in Section 2.2, where $2 n=2 \times 2^{m^{\star}}$ independent of $d$.

\subsection{Improvement}

When $\underline{S}_{u}$ is small, it usually becomes harder to estimate. For this reason, we consider the use of a new estimator to evaluate small first-order Sobol' indices in Variant $A$. This estimator called "Correlation 2" has been introduced by Owen in [14]. In this article, he discussed and highlighted the efficiency of "Correlation 2" when estimating small first-order indices. Our aim is to show that the use of "Correlation 2" in Variant $A$ may reduce the total number of model evaluations. This new estimator is,

$\widehat{\tau_{u}^{2}}=\frac{1}{n} \sum_{i=0}^{n-1}\left(f\left(\mathbf{x}_{i}\right)-f\left(\mathbf{z}_{i, u}: \mathbf{x}_{i,-u}\right)\right)\left(f\left(\mathbf{x}_{i, u}: \mathbf{x}_{i,-u}^{\prime}\right)-f\left(\mathbf{x}_{i}^{\prime}\right)\right)$,

where $\left(\mathbf{x}_{i}, \mathbf{x}^{\prime}{ }_{i}, \mathbf{z}_{i}\right) \stackrel{\text { iid }}{\sim} \mathscr{U}([0,1])^{3 d}$. It requires an additional set of $n$ model evaluations to estimate $\tau_{u}^{2}$.

We discuss below the potential improvement brought by using "Correlation 2" in Variant $A$. The idea is to replace the current estimator (5) by "Correlation 2" for each small first-order index

Assume that the number of small first-order indices is known and equals $\gamma$. We denote by $u_{1}, \ldots, u_{\gamma}$ the indices of the corresponding inputs and $\Gamma=\{1, \ldots, \gamma\}$. The cost of Variant $A$ including "Correlation 2" becomes,

$\sum_{j \in \Gamma} 2^{m_{u_{j}}^{\prime \prime}}+\sum_{j \in \Gamma} 2^{m_{u_{j}}^{\prime}}+\sum_{j \in \mathscr{D} \backslash \Gamma} 2^{m_{u_{j}}}+2 \times 2^{m^{\star}}$,

where:

- for $j \in \Gamma, 2^{m_{u_{j}}^{\prime \prime}}$ is the number of evaluations $f\left(\mathbf{z}_{i, u_{j}}: \mathbf{x}_{i,-u_{j}}\right)$ to estimate $\underline{S}_{u_{j}}$,

- for $j \in \Gamma, 2^{m_{u_{j}}^{\prime}}$ is the number of evaluations $f\left(\mathbf{x}_{i, u_{j}}: \mathbf{x}_{i,-u_{j}}^{\prime}\right)$ to estimate both $\underline{S}_{u_{j}}$ and $\bar{S}_{u_{j}}$,

- likewise, for $j \in \mathscr{D} \backslash \Gamma, 2^{m_{u_{j}}}$ is the number of evaluations $f\left(\mathbf{x}_{i, u_{j}}: \mathbf{x}_{i,-u_{j}}^{\prime}\right)$ to estimate both $\underline{S}_{u_{j}}$ and $\bar{S}_{u_{j}}$,

- $2 \times 2^{m^{\star}}$ is the number of evaluations $f\left(\mathbf{x}_{i}\right)$ and $f\left(\mathbf{x}_{i}^{\prime}\right)$ used in the estimation of each first-order and total effect index. 
We recall the cost of Variant $A$ without "Correlation 2",

$\sum_{j \in \Gamma} 2^{m_{u_{j}}}+\sum_{j \in \mathscr{D} \backslash \Gamma} 2^{m_{u_{j}}}+2 \times 2^{m^{\star}}$.

The difference $(17)-(18)$ equals:

$\sum_{j \in \Gamma} 2^{m_{u_{j}}}\left(2^{m_{u_{j}}^{\prime \prime}-m_{u_{j}}}+2^{m_{u_{j}}^{\prime}-m_{u_{j}}}-1\right)=\sum_{j \in \Gamma} c_{j}$.

Hence, the sign of this difference indicates whether or not using "Correlation 2" brings an improvement to Variant $A$. We distinguish two cases :

1) for $j \in \Gamma$, if the total effect index $\bar{S}_{u_{j}}$ requires as much or more evaluations than the first-order index $\underline{S}_{u_{j}}$. Since the total effect estimator is the same, as a consequence we have $m_{u_{j}}^{\prime}=m_{u_{j}}$ and $c_{j}>0$.

2) for $j \in \Gamma$, if the total effect index $\bar{S}_{u_{j}}$ requires less evaluations than the first-order index $\underline{S}_{u_{j}}$. In this case, if both $m_{u_{j}}^{\prime \prime}<m_{u_{j}}$ and $m_{u_{j}}^{\prime}<m_{u_{j}}$ then $c_{j} \leqslant 0$.

Overall we expect to observe case 2) more often than case 1). Indeed, the numerator of $\underline{S}_{u}$ requires to estimate $2 d$ dimensional integrals against only $d+1$ dimensional integrals for the numerator of $\bar{S}_{u}$. Hence, it seems reasonable to expect that it will take less points to estimate $\bar{S}_{u}$ than $\underline{S}_{u}$.

Furthermore, in case 2), we expect the two conditions $m_{u_{j}}^{\prime \prime}<m_{u_{j}}$ and $m_{u_{j}}^{\prime}<m_{u_{j}}$ to usually hold as in [14], "Correlation 2 " is shown to perform better for small first-order indices. To support this discussion, numerical examples are presented in Section 5.

In practice, one does not know which are the small Sobol' indices $\left(u_{1}, \ldots, u_{\gamma}\right)$. To overcome this issue, we propose the following alternative for Variant $A$. If at the end of the first iteration $\underline{S}_{u}$ is smaller than a threshold (in our case 0.1 , as suggested by Owen), then the estimator (5) is switched to "Correlation 2" for this particular $u$ and a third Sobol' sequence $\mathscr{P}^{\prime \prime}{ }_{m}=\left\{\mathbf{z}_{i}\right\}_{i=0}^{2^{m}-1}$ is constructed to obtain the corresponding evaluations $f\left(\mathbf{z}_{i, u}: \mathbf{x}_{i,-u}\right)$.

\section{Applications}

We illustrate our sequential estimation procedure with two classical test functions and one real example. In each case, Sobol' indices are estimated with the following three variants:

- Variant A.a as Variant $A$ without using "Correlation 2".

- Variant $A . b$ as Variant $A$ using "Correlation 2".

- Variant $B$.

The threshold to decide whether or not a first-order index is small is set to 0.1 .

For the two test functions, results of the three variants are compared based on the true estimation errors. These errors correspond to the absolute difference between the true values and their estimates:

$$
\delta_{\underline{S}_{u}}=\left|\underline{S}_{u}-\underline{S}_{u}\right|, \quad \delta_{\bar{S}_{u}}=\left|\bar{S}_{u}-\widehat{\bar{S}}_{u}\right| .
$$

We also compare the total number of evaluations of each variant. Results are averaged over 100 repetitions using Owen's scrambled Sobol' sequences [5,13]. For each repetition, we fix the tolerance $\varepsilon=5.10^{-3}$, set $\ell_{*}=5$, and $r=4$ (the algorithm starts with 512 Sobol' points). The algorithm error bound factor described in (14) is set to $\mathfrak{C}(m)=10 \times 2^{-m}$.

The failure rates $r_{\delta_{S_{u}}}$ and $r_{\delta_{\bar{S}_{u}}}$ of each estimate are evaluated over the 100 repetitions. These rates are the proportion of the repetitions in which the true estimation errors failed to satisfy $\delta_{\underline{S}_{u}} \leqslant \varepsilon$ and $\delta_{\underline{S}_{u}} \leqslant \varepsilon$. When $\delta_{\underline{S}_{u}}>\varepsilon$ and $\delta_{\underline{S}_{u}}>\varepsilon$, we can infer that our algorithm parameters that define the cone are not conservative enough and the integrands fall outside the cone (13).

\subsection{Classical test functions}

The two classical test functions considered in this article are the g-function introduced by Sobol' [17], and the function introduced by Bratley et al. [1]. The idea is to test our method over two categories of functions: additive (Bratley et al. function) and multiplicative (g-function).

\subsubsection{Sobol' g-function}

The g-function is defined as follows:

$$
f(\mathbf{x})=\prod_{j=1}^{d} g_{j}\left(x_{j}\right), \quad g_{j}\left(x_{j}\right)=\frac{\left|4 x_{j}-2\right|+a_{j}}{1+a_{j}}, a_{j} \geqslant 0 .
$$

Each value $a_{j}$ determines the relative importance of the $x_{j}$. When the value of $a_{j}$ gets closer to zero the variable $x_{j}$ becomes more influent. For this example, we chose $d=6$ and $a_{1}=0, a_{2}=0.5, a_{3}=3, a_{4}=9, a_{5}=99$, and $a_{6}=99$.

Table 1 shows the averaged estimation errors obtained with Variant A.a as well as the averaged total number of evaluations performed. Table 2 shows the same results obtained with Variant $A . b$.

Table 1 Averaged estimation errors $\delta_{\underline{S}_{u}}, \delta_{\bar{S}_{u}}$ and total number of evaluations for Variant A.a.

\begin{tabular}{ccccccc}
\hline input & $\underline{S}_{u}$ & $\delta_{\underline{S}_{u}}$ & $r_{\delta_{\underline{S}_{u}}}$ & $\bar{S}_{u}$ & $\delta_{\bar{S}_{u}}$ & $r_{\delta_{\bar{S}_{u}}}$ \\
\hline$x_{1}$ & 0.5868 & 0.0004 & 0.02 & 0.6901 & 0.0005 & 0.02 \\
\hline$x_{2}$ & 0.2608 & 0.0006 & 0.02 & 0.3562 & 0.0004 & 0 \\
\hline$x_{3}$ & 0.0367 & 0.0011 & 0.03 & 0.0563 & 0.0008 & 0 \\
\hline$x_{4}$ & 0.0058 & 0.0012 & 0.02 & 0.0092 & 0.0003 & 0 \\
\hline$x_{5}$ & $5.10^{-5}$ & 0.0003 & 0 & $9.10^{-5}$ & $<10^{-4}$ & 0 \\
\hline$x_{6}$ & $5.10^{-5}$ & 0.0003 & 0 & $9.10^{-5}$ & $<10^{-4}$ & 0 \\
\hline \hline
\end{tabular}

Total number of evaluations: 63088 
Table 2 Averaged estimation errors $\delta_{\underline{S}_{u}}, \delta_{\bar{S}_{u}}$ and total number of evaluations for Variant $A . b$.

\begin{tabular}{ccccccc}
\hline input & $\underline{S}_{u}$ & $\delta_{\underline{S}_{u}}$ & $r_{\delta_{\underline{S}_{u}}}$ & $\bar{S}_{u}$ & $\delta_{\bar{S}_{u}}$ & $r_{\delta_{\bar{S}_{u}}}$ \\
\hline$x_{1}$ & 0.5868 & 0.0005 & 0.03 & 0.6901 & 0.0006 & 0.02 \\
\hline$x_{2}$ & 0.2608 & 0.0007 & 0.03 & 0.3562 & 0.0003 & 0 \\
\hline$x_{3}$ & 0.0367 & 0.0015 & 0.05 & 0.0563 & 0.0009 & 0 \\
\hline$x_{4}$ & 0.0058 & 0.0003 & 0 & 0.0092 & 0.0003 & 0 \\
\hline$x_{5}$ & $5.10^{-5}$ & $<10^{-4}$ & 0 & $9.10^{-5}$ & $<10^{-4}$ & 0 \\
\hline$x_{6}$ & $5.10^{-5}$ & $<10^{-4}$ & 0 & $9.10^{-5}$ & $<10^{-4}$ & 0 \\
\hline \hline
\end{tabular}

Total number of evaluations: 62703

The main observation is that both approaches give similar results both in terms of estimation errors, failure rates and total number of evaluations. The use of "Correlation 2" in Variant $A . b$ to estimate the four small first-order Sobol' indices $\underline{S}_{3}, \underline{S}_{4}, \underline{S}_{5}, \underline{S}_{6}$ does not seem to bring much improvement. We propose to draw boxplots of the estimation errors to further investigate the results.

Figure 2 shows boxplots of the 100 estimation errors $\delta_{\underline{S}_{u}}$ obtained with both Variant A. $a$ and Variant $A . b$ for the four inputs $x_{3}, x_{4}, x_{5}, x_{6}$. The dashed horizontal line marks the tolerance $\varepsilon=5.10^{-3}$.

Fig. 2 Boxplots of estimation errors $\delta_{S}$ for the four inputs $x_{3}, x_{4}, x_{5}, x_{6}$ obtained with both Variant $A . a$ and Variant A.b. The dashed horizontal line marks the tolerance $\varepsilon=5.10^{-3}$.

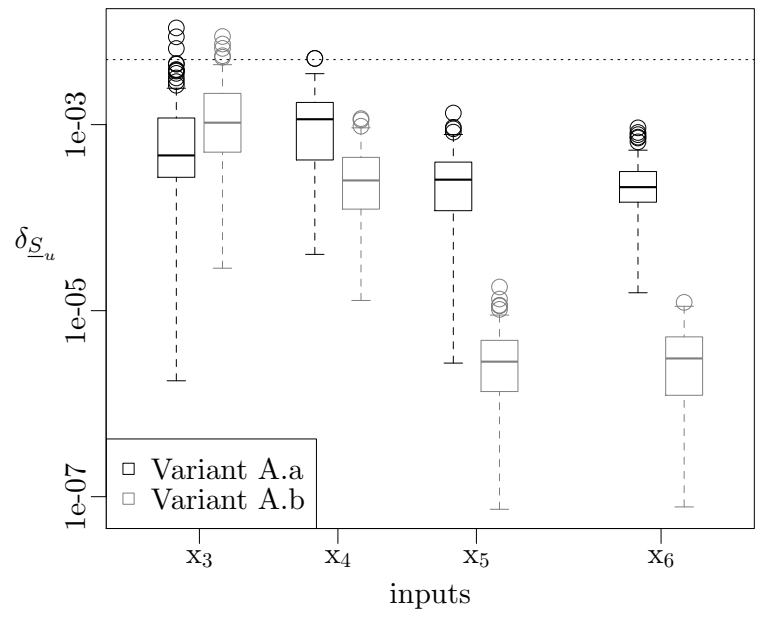

As expected, we observe that the use of "Correlation 2" in Variant A.b results in lower estimation errors for these four inputs. The discrepancy observed does not stand out in Table 2 due to its low magnitude $\left(10^{-4} \sim 10^{-5}\right)$.

Table 3 shows averaged estimation errors $\delta_{\underline{S}_{u}}$ and total number of evaluations for Variant $B$. The results show that the use of Variant $B$ leads to slightly higher estimation errors and higher failure rates than those obtained with Variant $A . a$
Table 3 Averaged estimation errors $\delta_{\underline{S}_{u}}$ and total number of evaluations for Variant $B$.

\begin{tabular}{cccc}
\hline input & $\underline{S}_{u}$ & $\delta_{\underline{S}_{u}}$ & $r_{\delta_{\underline{S}_{u}}}$ \\
\hline$x_{1}$ & 0.5868 & 0.0011 & 0.07 \\
\hline$x_{2}$ & 0.2608 & 0.0013 & 0.08 \\
\hline$x_{3}$ & 0.0367 & 0.0018 & 0.09 \\
\hline$x_{4}$ & 0.0058 & 0.0013 & 0.1 \\
\hline$x_{5}$ & $5.10^{-5}$ & 0.0031 & 0.04 \\
\hline$x_{6}$ & $5.10^{-5}$ & 0.0036 & 0.05 \\
\hline \multicolumn{4}{l}{ Total number of evaluations: 32768}
\end{tabular}

or Variant A.b. However, the total number of evaluations is twice as small. As such, this approach remains interesting when one wants to estimate only first-order indices.

\subsubsection{Bratley et al. function}

In this second example, we consider the Bratley et al. function defined by,

$$
f\left(x_{1}, \ldots, x_{d}\right)=\sum_{i=1}^{d}(-1)^{i} \prod_{j=1}^{i} x_{j} .
$$

The importance of each variable $x_{j}$ depends on their own rank. More explicitly, $x_{1}$ is more influent than $x_{2}$ that is respectively more influent than $x_{3}$ and so on.

As for the g-function, Tables 4 and 5 show averaged estimation errors and total number of evaluations for Variant A. $a$ and Variant A.b.

Table 4 Averaged estimation errors $\delta_{\underline{S}_{u}}, \delta_{\bar{S}_{u}}$ and total number of evaluations for Variant A.a.

\begin{tabular}{ccccccc}
\hline input & $\underline{S}_{u}$ & $\delta_{\underline{S}_{u}}$ & $r_{{\underline{S_{u}}}_{u}}$ & $\bar{S}_{u}$ & $\delta_{\bar{S}_{u}}$ & $r_{\delta_{\bar{S}_{u}}}$ \\
\hline$x_{1}$ & 0.6529 & 0.0004 & 0 & 0.7396 & 0.0003 & 0. \\
\hline$x_{2}$ & 0.1791 & 0.0006 & 0 & 0.2659 & 0.0004 & 0 \\
\hline$x_{3}$ & 0.0370 & 0.0058 & 0.71 & 0.0764 & 0.0012 & 0.02 \\
\hline$x_{4}$ & 0.0133 & 0.0022 & 0.12 & 0.0343 & 0.0020 & 0.07 \\
\hline$x_{5}$ & 0.0015 & 0.0024 & 0.11 & 0.0062 & 0.0014 & 0 \\
\hline$x_{6}$ & 0.0015 & 0.0021 & 0.06 & 0.0062 & 0.0010 & 0.03 \\
\hline \multicolumn{7}{l}{ Total number of evaluations: 70129} \\
\hline
\end{tabular}

Variant $A . b$ gives lower estimation errors and fewer failure rates than Variant A. $a$ on the four small first-indices $\underline{S}_{3}$, $\underline{S}_{4}, \underline{S}_{5}, \underline{S}_{6}$ which highlights the performance of "Correlation 2 ". The discrepancy is particularly notable for input $x_{3}$ where Variant $A$. $a$ reaches a failure rate of $71 \%$ against only $4 \%$ for Variant $A . b$. Furthermore, Variant $A . b$ requires less evaluations than Variant A.a. The boxplots represented in Figure 3 emphasize the latter observations.

Table 6 shows averaged estimation errors $\delta_{\underline{S}_{u}}$ and total number of evaluations for Variant $B$. 
Table 5 Averaged estimation errors $\delta_{\underline{S}_{u}}, \delta_{\bar{S}_{u}}$ and total number of evaluations for Variant $A . b$.

\begin{tabular}{ccccccc}
\hline input & $\underline{S}_{u}$ & $\delta_{\underline{S}_{u}}$ & $r_{\delta_{\underline{S}_{u}}}$ & $\bar{S}_{u}$ & $\delta_{\bar{S}_{u}}$ & $r_{\delta_{\bar{S}_{u}}}$ \\
\hline$x_{1}$ & 0.6529 & 0.0004 & 0 & 0.7396 & 0.0003 & 0 \\
\hline$x_{2}$ & 0.1791 & 0.0006 & 0 & 0.2659 & 0.0005 & 0 \\
\hline$x_{3}$ & 0.0370 & 0.0019 & 0.04 & 0.0764 & 0.0013 & 0.01 \\
\hline$x_{4}$ & 0.0133 & 0.0016 & 0.01 & 0.0343 & 0.0017 & 0.02 \\
\hline$x_{5}$ & 0.0015 & 0.0003 & 0 & 0.0062 & 0.0015 & 0.04 \\
\hline$x_{6}$ & 0.0015 & 0.0004 & 0 & 0.0062 & 0.0011 & 0 \\
\hline \hline
\end{tabular}

Total number of evaluations: 68045

Fig. 3 Boxplots of estimation errors $\delta_{S_{u}}$ for the four inputs $x_{3}, x_{4}, x_{5}, x_{6}$ obtained with both Variant A.a and Variant A.b. The dashed horizontal line marks the tolerance $\varepsilon=5.10^{-3}$.

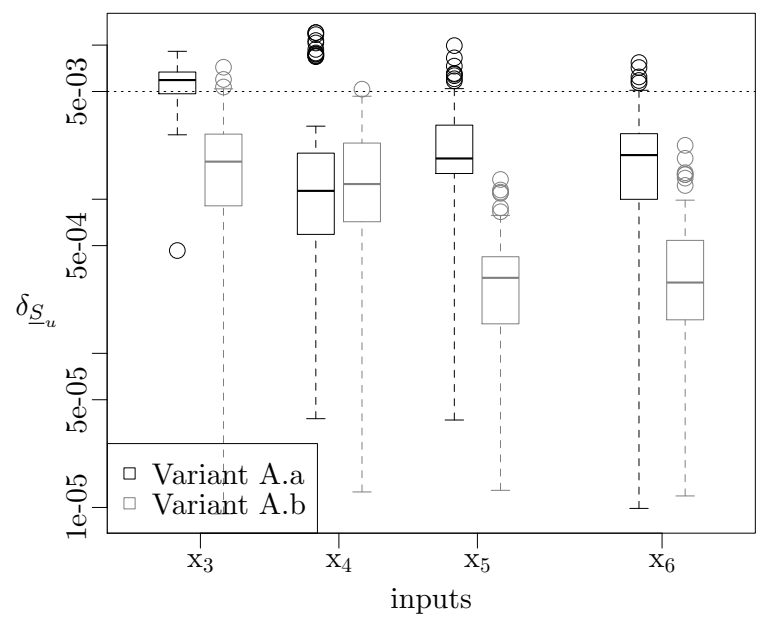

Table 6 Averaged estimation errors $\delta_{\underline{S}_{u}}$ and total number of evaluations for Variant $B$.

\begin{tabular}{cccc}
\hline input & $\underline{S}_{u}$ & $\delta_{\underline{S}_{u}}$ & $r_{{\underline{S_{\underline{u}}}}_{\underline{u}_{u}}}$ \\
\hline$x_{1}$ & 0.6529 & 0.0003 & 0 \\
\hline$x_{2}$ & 0.1791 & 0.0006 & 0 \\
\hline$x_{3}$ & 0.0370 & 0.0010 & 0 \\
\hline$x_{4}$ & 0.0133 & 0.0038 & 0.25 \\
\hline$x_{5}$ & 0.0015 & 0.0024 & 0 \\
\hline$x_{6}$ & 0.0015 & 0.0026 & 0 \\
\hline \hline \multicolumn{4}{l}{ Total number of evaluations: 65536}
\end{tabular}

The estimation errors are lower than those of Variant A.a but higher than those of Variant $A . b$. Failure rates are similar to those of Variant A.b. Since the total number of evaluations is close to the one of Variant $A . b$, the conclusion is that Variant $B$ does not bring much improvement for this example.

From the results on these two test functions, the main conclusion is that Variant $A . b$ performs the best. This highlights the efficiency of "Correlation 2" to estimate small first-order indices with quasi-Monte Carlo methods. Even if
"Correlation 2" originally requires more model evaluations, this drawback is completely overridden when this estimator is included into a sequential procedure such as ours. For the case where we are only interested in estimating first-order indices, Variant $B$ should be the best choice as illustrated with the g-function.

\subsection{Real case model}

As a real case example, we will study the payoff function of an arithmetic mean Asian call option. For a discretized Brownian motion $\mathbf{B}=\left(B_{t_{1}}, B_{t_{2}}, \ldots, B_{t_{d}}\right)$ at times $t_{i}=T \times i / d$, the payoff of the option is:

$f_{\text {payoff }}(\mathbf{B})=\mathrm{e}^{-\rho T} \max \left(\frac{1}{d} \sum_{i=1}^{d} S_{0} \mathrm{e}^{\left(\rho-\sigma^{2} / 2\right) t_{i}+\sigma B_{t_{i}}}-K, 0\right)$

where $T$ is the maturity of the option, $S_{0}$ is the initial stock price, $\sigma$ the volatility of the stock, $\rho$ the interest rate, and $K$ the strike price.

The discretized Brownian motion $\mathbf{B}$ follows a multivariate normal distribution with mean $\mathbf{0}$ and covariance matrix $\Sigma$, with $\Sigma_{i j}=\min \left(t_{i}, t_{j}\right)$. If $\mathbf{Y} \sim N\left(\mathbf{0}, I_{d}\right)$, then for any matrix $M, M \mathbf{Y} \sim N(\mathbf{0}, \Sigma)$ where $\Sigma=M M^{t}$. Hence, we can generate the discretized Brownian motion using the inverse normal cumulative distribution function $\phi^{-1}: \mathbf{B} \sim M \phi^{-1}(\mathbf{X})$ with $\phi^{-1}(\mathbf{X})=\left(\phi^{-1}\left(X_{1}\right), \ldots, \phi^{-1}\left(X_{d}\right)\right)^{t}$ and $\mathbf{X} \sim \mathscr{U}\left([0,1]^{d}\right)$. Thus, we can estimate the Sobol' indices over $f_{\text {payoff }}\left(M \phi^{-1}(\mathbf{X})\right)$. In this particular application, the choice of $M$ is pivotal to reduce the effective dimensionality of the model. In this regard, Sobol' indices can help us assess whether we reduce the effective dimensionality. We will compare the case with either the Cholesky decomposition or the PCA construction.

We propose to analyze the results with averaged Sobol' estimates and averaged total number of evaluations over 100 repetitions. The tolerance $\varepsilon$ and parameters $\ell^{*}, r$ and $\mathfrak{C}(m)$ are set as previously.

Tables 7, 8, and 9 show the results when $M$ corresponds to the Cholesky decomposition of $\Sigma$.

Table 7 Averaged values of $\underline{\widehat{S}}_{u}, \hat{\bar{S}}_{u}$, and total number of evaluations of Variant A.a with Cholesky decomposition.

\begin{tabular}{ccc}
\hline input & $\hat{\underline{S}}_{u}$ & $\widehat{\bar{S}}_{u}$ \\
\hline$x_{1}$ & 0.2120 & 0.5650 \\
\hline$x_{2}$ & 0.1370 & 0.4420 \\
\hline$x_{3}$ & 0.0820 & 0.3150 \\
\hline$x_{4}$ & 0.0408 & 0.1950 \\
\hline$x_{5}$ & 0.0142 & 0.0944 \\
\hline$x_{6}$ & 0.0048 & 0.0257 \\
\hline \hline \multicolumn{3}{l}{ Total number of evaluations: 648821} \\
\hline
\end{tabular}


Table 8 Averaged values of $\widehat{S}_{u}, \hat{\bar{S}}_{u}$ and total number of evaluations of Variant $A . b$ with Cholesky decomposition.

\begin{tabular}{ccc}
\hline input & $\hat{\underline{S}}_{u}$ & $\hat{\bar{S}}_{u}$ \\
\hline$x_{1}$ & 0.2120 & 0.5650 \\
\hline$x_{2}$ & 0.1370 & 0.4420 \\
\hline$x_{3}$ & 0.0822 & 0.3150 \\
\hline$x_{4}$ & 0.0434 & 0.1950 \\
\hline$x_{5}$ & 0.0169 & 0.0944 \\
\hline$x_{6}$ & 0.0049 & 0.0259 \\
\hline \hline \multicolumn{3}{l}{ Total number of evaluations: 699487}
\end{tabular}

Table 9 Averaged values of $\widehat{\underline{S}}_{u}$ and total number of evaluations of Variant $B$ with Cholesky decomposition.

\begin{tabular}{ll}
\hline input & $\underline{\hat{S}}_{u}$ \\
\hline$x_{1}$ & 0.2120 \\
\hline$x_{2}$ & 0.1350 \\
\hline$x_{3}$ & 0.0774 \\
\hline$x_{4}$ & 0.0338 \\
\hline$x_{5}$ & 0.0149 \\
\hline$x_{6}$ & 0.0028 \\
\hline \hline
\end{tabular}

It turns out that Variant $A . b$ does not perform better than Variant A.a. Averaged Sobol' estimates are similar but the average total number of model evaluations is slightly worse. The explanation is that total effect Sobol' indices require more evaluations than their corresponding first-order indices. As such, "Correlation 2" falls into case 1) explained in Section 4.2. Variant $B$ shows a drastic improvement on the total number of evaluations with the drawback of a false estimation for input $x_{4}$. Its discrepancy is higher than $\varepsilon$ when comparing to the other two variants.

Tables 10, 11, and 12 show the results when $M$ corresponds to the PCA decomposition of $\Sigma$.

Table 10 Averaged values of $\underline{\underline{S}}_{u}, \hat{\bar{S}}_{u}$ and total number of evaluations of Variant A. $a$ with PCA decomposition.

\begin{tabular}{ccc}
\hline input & $\hat{\widehat{S}}_{u}$ & $\widehat{\bar{S}}_{u}$ \\
\hline$x_{1}$ & 0.9800 & 0.9960 \\
\hline$x_{2}$ & 0.0037 & 0.0172 \\
\hline$x_{3}$ & 0.0016 & 0.0023 \\
\hline$x_{4}$ & 0.0014 & 0.0005 \\
\hline$x_{5}$ & 0.0005 & 0.0001 \\
\hline$x_{6}$ & 0.0002 & 0.0000 \\
\hline \multicolumn{3}{l}{ Total number of evaluations: 396221}
\end{tabular}

Variant $A . a$ performs as well as Variant $A . b$ in terms of average total number of evaluations. However, Variant A.a is most probably giving worse estimates for the small first-
Table 11 Averaged values of $\widehat{S}_{u}, \hat{\bar{S}}_{u}$ and total number of evaluations of Variant $A . b$ with PCA decomposition.

\begin{tabular}{ccc}
\hline input & $\hat{\underline{S}}_{u}$ & $\hat{\bar{S}}_{u}$ \\
\hline$x_{1}$ & 0.9800 & 0.9960 \\
\hline$x_{2}$ & 0.0034 & 0.0172 \\
\hline$x_{3}$ & 0.0004 & 0.0023 \\
\hline$x_{4}$ & 0.0001 & 0.0005 \\
\hline$x_{5}$ & 0.0000 & 0.0001 \\
\hline$x_{6}$ & 0.0000 & 0.0000 \\
\hline \multicolumn{3}{l}{ Total number of evaluations: 398648}
\end{tabular}

Table 12 Averaged values of $\underline{\underline{S}}_{u}$ and total number of evaluations of Variant $B$ with PCA decomposition.

\begin{tabular}{cc}
\hline input & $\underline{\underline{S}}_{u}$ \\
\hline$x_{1}$ & 0.9800 \\
\hline$x_{2}$ & 0.0037 \\
\hline$x_{3}$ & 0.0028 \\
\hline$x_{4}$ & 0.0029 \\
\hline$x_{5}$ & 0.0017 \\
\hline$x_{6}$ & 0.0029 \\
\hline \multicolumn{2}{l}{ Total number of evaluations: 262 144 }
\end{tabular}

order indices. Table 10 shows that averaged $\underline{S}_{4}$ is greater than $\widehat{\bar{S}}_{4}, \widehat{S}_{5}$ greater than $\widehat{\bar{S}}_{5}$, and $\widehat{S}_{6}$ greater than $\hat{\bar{S}}_{6}$, which is inconsistent with the property $\underline{S}_{u} \leqslant \bar{S}_{u}$. Once again, variant $B$ shows an improvement on the total number of evaluations with the drawback of slightly overestimating the small firstorder indices.

Overall, each variant captures well how the PCA Brownian motion construction reduces the high effective dimensionality of the payoff function, in contrast to the initial Cholesky decomposition.

\section{Conclusion}

When estimating Sobol' indices, the question of how many evaluations must be performed to reach a desired precision is often raised by practitioners. This question is difficult to address mostly because the number of evaluations needed depends on the complexity of the model studied. As such, it is hard to bring out a general rule of thumb.

The sequential estimation procedure proposed in this article offers a practical solution with the construction of an estimator and error bound for Sobol' indices. The number of points is progressively augmented until the error bound becomes lower than a user specified tolerance. The procedure presented combines Sobol' sequences with either Saltelli's strategy to estimate both first-order and total effect indices, or the replication procedure to estimate only first-order indices. Furthermore, we investigated the use of a recent es- 
timator well-suited to the estimation of small first-order indices using quasi-Monte Carlo methods. The efficiency of this estimator, called "Correlation 2", was assessed and highlighted on two test functions. Overall, the variant combining Saltelli's strategy and "Correlation 2" gave the best results, with low failure rates across all indices.

As a future project, the same estimators and algorithms can be designed for rank-1 lattices using the results in [8], and noticing that rank-1 lattices of the same size are also replicated designs of order 1 .

Acknowledgements The authors thank Fred J. Hickernell and Clémentine Prieur for initiating this collaborative work and Elise Arnaud for her proofreading. The authors are grateful to Stephen Joe, Frances Y. Kuo and Art B. Owen for their helpful answers and suggestions.

\section{References}

1. Bratley, P., Fox, B.L., Niederreiter, H.: Implementation and tests of low-discrepancy sequences, ACM Trans. Model. Comput. Simul. 2(3), 195-213 (1992)

2. Gilquin, L., Arnaud, E., Monod, H., Prieur, C.: Recursive estimation procedure of Sobol' indices based on replicated designs, preprint available at https://hal.inria.fr/hal-01291769, (2016).

3. Gilquin, L., Jiménez Rugama, Ll.A., Arnaud, E., Hickernell, F.J., Monod, H., Prieur, C.: Iterative construction of replicated designs based on Sobol' sequences, preprint available at https://hal. inria.fr/hal-01349444 (2016)

4. Hickernell, F.J., Jiménez Rugama, Ll.A.: Reliable Adaptative Cubature Using Digital Sequences: Monte Carlo and Quasi-Monte Carlo Methods, vol. 163, 367-383 (2016)

5. H.S. Hong, F.J. Hickernell.: Algorithm 823: Implementing scrambled digital nets, ACM Trans. Math. Software, vol. 29, 95-109, (2003)

6. Hoeffding, W.F.: A class of statistics with asymptotically normal distribution, Ann. Math. Stat. 19(3), 293-325 (1948)

7. Janon, A., Klein, T., Lagnoux A., Nodet, M., Prieur C.: Asymptotic normality and efficiency of two Sobol' index estimators, ESAIM Probab. Stat. 18, 342-364 (2014)

8. Jiménez Rugama, Ll.A., Hickernell, F.J.: Adaptive Multidimensional Integration Based on Rank-1 Lattices: Monte Carlo and Quasi-Monte Carlo Methods, vol. 163, 407-422 (2016)

9. Lemieux,C.: Monte Carlo and Quasi-Monte Carlo Sampling, Springer, New-York (2009)

10. Mara, T.A., Rakoto Joseph, 0.: Comparison of some efficient methods to evaluate the main effect of computer model factors, J. Statist. Comput. Simulation 78(2), 167-178 (2008)

11. McKay, M.D.: Evaluating prediction uncertainty, Los Alamos National Laboratory Report NUREG/CR- 6311, LA-12915-MS. (1995)

12. Niederreiter, H.: Random Number Generation and Quasi-Monte Carlo Methods: CBMS-NSF Regional Conference Series in Applied Math., vol. 63, SIAM, Philadelphia (1992)

13. A.B. Owen.: Randomly permuted $(t, m, s)$-nets and $(t, s)$ sequences: Monte Carlo and Quasi-Monte Carlo Methods in Scientific Computing, vol. 106, 299-317 (1995)

14. Owen, A.B.: Better estimation of small Sobol' sensitivity indices, ACM Trans. Model. Comput. Simul. 23(2), :11 (2013)

15. Saltelli, A.: Making best use of models evaluations to compute sensitivity indices, Comput. Phys. Commun. 145(2), 280-297 (2002)
16. Sobol', I.M.: On the distribution of points in a cube and the approximate evaluation of integrals, USSR Comput. Math. Math. Phys. 7(4), 86-112 (1967)

17. Sobol', I.M.: Sensitivity indices for nonlinear mathematical models, Mathematical Modeling and Computational Experiment 1, 407-414 (1993)

18. Tissot, J.Y., Prieur, C.: A randomized Orthogonal Array-based procedure for the estimation of first- and second-order Sobol' indices, J. Statist. Comput. Simulation 85(7), 1358-1381 (2015)

19. Tong C.: Self-validated variance-based methods for sensitivity analysis of model outputs, Reliab. Eng. Syst. Saf. 95(3), 301-309 (2010) 